\title{
Wnt/ $\beta$-catenin Inhibitor ICG-001 Enhances the Antitumor Efficacy of Radiotherapy by Increasing Radiation-induced DNA Damage and Improving Tumor Immune Microenvironment in Hepatocellular Carcinoma
}

\section{Yan Huang}

Southern Medical University Nanfang Hospital

\section{Hailong Sheng}

Southern Medical University Nanfang Hospital

Yazhi Xiao

Southern Medical University Nanfang Hospital

Zhihong Zhang

Southern Medical University Nanfang Hospital

Yiyao Chen

Southern Medical University Nanfang Hospital

Zhenru Zhu

Southern Medical University Nanfang Hospital

Chuanhui Cao

Southern Medical University Nanfang Hospital

Jingyuan Sun ( $D$ 15013050515@163.com )

Southern Medical University Nanfang Hospital

\section{Research}

Keywords: Hepatocellular carcinoma, Wnt/ $\beta$-catenin inhibitor, Radiotherapy, Radioresistance, Tumor immune microenvironment, cGAS/STING

Posted Date: August 14th, 2020

DOI: https://doi.org/10.21203/rs.3.rs-57294/v1

License: (c) (1) This work is licensed under a Creative Commons Attribution 4.0 International License.

Read Full License 
Version of Record: A version of this preprint was published at Radiotherapy and Oncology on September 1st, 2021. See the published version at https://doi.org/10.1016/j.radonc.2021.06.034. 


\section{Abstract}

Background: Radiotherapy has a promising anti-tumor effect in hepatocellular carcinoma (HCC), depending on the its regulatory effects on both cancer cells and tumor immune microenvironment (TME). Wnt/ $\beta$-catenin signaling pathway activation, which is one of the most common alterations in HCC patients, has been reported to induce radioresistance, and also create immunosuppressive TME. However, it is unclear whether inhibition of wnt/ $\beta$-catenin pathway could enhance the treatment efficacy of radiotherapy. In this study, we aim to explore the effect of wnt/ $\beta$-catenin inhibitor ICG-001 in combination with radiotherapy and the underlying mechanism in HCC.

Methods: C57BL/6 and nude mouse subcutaneous tumor models were used to evaluate the efficacy of different treatment regimens in tumor growth control, tumor recurrence inhibition and survival improvement. Flow cytometry was performed to assess the alterations of tumor infiltrating lymphocytes (TILs). Radioresistance was investigated by clone formation assay and $\mathrm{y}-\mathrm{H} 2 \mathrm{AX}$ measurements. Wnt/ $\beta$ catenin and cGAS/STING pathway activation was detected by immunoblotting.

Results: The addition of ICG-001 to radiotherapy exhibited better anti-tumor control efficacy in tumorbearing C57BL/ 6 mice than nude mice, which suggested that ICG-001 had a critical role in activating TME. The comprehensive analysis of TILs revealed that compared with radiotherapy alone, the combination of ICG-001 with radiotherapy boosted the infiltration and IFN-y production ability of TIL $\mathrm{CD}^{+} \mathrm{T}$ cells, meanwhile reduced the number of TIL Tregs. Moreover, mechanism study demonstrated that ICG-001 exerted a radiosensitizing effect on HCC cells, thus leading to stronger activation of cGAS/STING signaling pathway upon radiotherapy in vitro and in vivo. Utilization of STING inhibitor, C176 , significantly impaired the synergetic effect of ICG-001 with radiotherapy on tumor control and TME activation. Furthermore, combination therapy led to a stronger immunologic memory and lasting antitumor immunity than radiotherapy, thus preventing tumor relapse in HCC tumor-bearing mice.

Conclusion: Our findings showed that ICG-001 increased radioresistance and improved TME upon radiotherapy in HCC. Compared with radiotherapy alone, the combination of ICG-001 with radiotherapy displayed better therapeutic efficacy in inhibiting tumor growth, prolonging survival, and preventing recurrence in tumor-bearing mice. These data indicated that ICG-001 might be a potential synergetic treatment for radiotherapy and radioimmunotherapy in HCC.

\section{Background}

Worldwide, hepatocellular carcinoma (HCC) is the sixth most common incident cancer and the fourth leading cases of cancer-related death [1]. Surgical resection and liver transplantation are first-line treatments for early-staged HCC patients[2]. However, a significant proportion of HCC patients are late staged at diagnosis, for whom surgery is not a suitable option. Radiotherapy, especially stereotactic body radiation therapy (SBRT) has emerged as an effective noninvasive alternative and safe approaches for HCC local control and liver oligo-metastases[3, 4]. 
Radiotherapy was widely known to exert its cytotoxic role on cancer cells via radiation-induced DNA damage[5, 6]. Besides the tumor-cell autonomous effect, radiotherapy also has non-tumor cellautonomous aspects by leasing new antigens, meanwhile facilitating dsDNA (double-strand DNA) production and activating cGAS/STING signaling pathway, thus initiating antitumor immunity and improving tumor immune microenvironment (TME) $[7,8]$. Moreover, increasing preclinical studies have shown that the combination of radiotherapy and immunotherapy (radioimmunotherapy) improve the local control and recurrence of varied types of cancers[9-11]. These findings indicated radioimmunotherapy could be a potential effective therapeutic strategy for cancer treatment. Notably, what needs to be noticed that several studies have suggested that there are mutual effects between radiotherapy and immune system. For example, Fu et al. reported that therapeutic effects of radiotherapy on local tumor require $\mathrm{CD} 8^{+} \mathrm{T}$ cells[12]. Bai et al. revealed that inhibiting the infiltration of $\mathrm{CD} 8+\mathrm{T}$ cells in TME after radiotherapy leads to radioresistance in cancer cells [13]. Therefore, these evidences suggested that disrupting the reciprocal effect between radiation and TME might possibly become a promising approach for radioimmunotherapy.

Wnt/ $\beta$-catenin signaling pathway is well studied to be involved in development and progression of HCC $[14,15]$. Numerous studies have shown that wnt/ $\beta$-catenin signaling activation causes stronger DNA damage response (DDR) upon radiation, thus attenuating DNA damage and increasing cell survival, and as a result, leading to radioresistance of cancer cells $[16,17]$. Moreover, several preclinical studies have demonstrated that wnt/ $\beta$-catenin activation contributed to less infiltration of lymphocytes, which then created immunosuppressive microenvironment and decreased response to immunotherapy[18, 19]. In consistent with that, a next-generation sequencing analysis prospectively genotyped 127 advanced HCC patients revealed that wnt/ $\beta$-catenin activation was associated with innate resistant to immune checkpoint inhibitors (ICls), shown by lower disease control rate, shorter median progression free survival and overall survival upon ICls treatment[20]. Taken together, we assumed that wnt/ $\beta$-catenin signaling might play a critical roles on both increasing anti-tumor effect and activate TME in combination with radiotherapy. In addition, inhibiting wnt/ $\beta$-catenin signaling might impair the mutual effects between radiotherapy response and TME, thus could potentially increase the therapeutic efficacy of radiotherapy or radioimmunotherapy.

$\beta$-catenin is a core molecule in transducing wnt signaling by binding to LEF-1/TCF4 and promoting the transcription of targeted genes, including Jun, c-Myc and CyclinD1[21]. ICG-001 is a CBP/ $\beta$-catenin inhibitor, which impaired wnt/ $\beta$-catenin-mediated transcription. As the derivative of ICG-001, PRI-724 has entered early-phase clinical trial for advanced solid tumors (NCT01302405)[22]. In addition, PRI-724 was proved to be well-tolerated by patients with HCV cirrhosis[23]. In this study, we explore the synergistic antitumor and immune-editing role of ICG-001 in combination with radiotherapy. Our findings highlighted the potential of ICG-001 could be developed as a new synergistic treatment paradigm in combined with radiotherapy or radioimmunotherapy in HCC patients.

\section{Methods}




\section{Cell culture}

Hepa1-6 (C57BL/ 6 murine liver cancer cell line) and HCC-LM3 (Human hepatocellular cancer cell lines) cells were obtained from the Shanghai Institute of Biochemistry and Cell Biology. Cells were cultured in DMEM (Gibco, USA) supplemented with $10 \%$ fetal bovine serum (Gibco, USA). Both cells were cultured using standard procedures in a $37^{\circ} \mathrm{C}$ humidified incubator with $5 \% \mathrm{CO}_{2}$.

\section{Mice treatment}

C57BL/ 6 and nude mice (5 to 10 weeks of age) were obtained from the Animal Resource Centre (Southern Medical University, China) and the Guangdong Medical Laboratory Animal Center (Guangzhou, China). Mice were housed, fed, and monitored in accordance with protocols approved by the Committee for Animal Research at Southern Medical University (Guangzhou, China). Hepal-6 cells $\left(1 \times 10^{7}\right)$ were implanted subcutaneously in the right hind flank of the C57BL/ 6 or nude mice. Tumor volumes were calculated using a standard formula: length $\times$ width $^{2} / 2$. For radiotherapy experiment, radiation was administered when tumors reached approximately $200 \mathrm{~mm}^{3}$. Mice were anesthetized by intraperitoneal injection with $2 \%$ sodium pentobarbital solution and received three fractions of $6 \mathrm{~Gy}$ on days $1,3,5$ from the start of treatment. For ICG-001 experiment, mice were intraperitoneally injected with ICG-001 (S2662, Selleck; $20 \mathrm{mg} / \mathrm{kg}$ ) on days 1, 3, 5 from the start of treatment. For C-176 experiment, mice were intraperitoneally injected with C-176 (S6575, Selleck; $5 \mathrm{mg} / \mathrm{kg} / \mathrm{d}$ ) daily from one week before radiation till the complete of radiation.

\section{Lymphocytes extraction}

The mice were euthanized to harvest the tumors and spleens. The tumor tissue was immediately submerged in RPMI media with $0.5 \mathrm{mg} / \mathrm{ml}$ collagenase IV (Solarbio Life Sciences) and $0.1 \mathrm{mg} / \mathrm{ml}$ deoxyribonuclease type I (Solarbio Life Sciences) at $37^{\circ} \mathrm{C}$ for 1 hour, and then was dissociated. The spleen was harvested and directly mechanically dissociated on a frosted glass slide to prepare for the cell suspension. The single-cell suspension of tumor or spleen was obtained by filtration using a 70- $\mu \mathrm{m}$ cell strainer (Corning Inc.), followed by erythrocyte elimination by lysis in $1 \mathrm{ml}$ erythrocyte lysis buffer (150 $\mathrm{mM} \mathrm{NH}_{4} \mathrm{Cl}, 10 \mathrm{mM} \mathrm{NaHCO}_{3}, 0.1 \mathrm{mM}$ EDTA) for 1 minute at room temperature The single-cell suspension was counted and at least $1 \times 10^{6}$ cells were prepared for subsequent experiments. For detection of IFN- $\gamma$, cells were incubated with Leuko Act Cktl With GolgiPlug (550583, BD Biosciences) at $37^{\circ} \mathrm{C}$ for $4-6$ hours, and were further cultured for 2 hours for subsequent staining.

\section{Flow cytometry analysis}

Cells were stained with antibodies for 30 minutes on ice. After permeabilization with Transcription Factor Buffer Set (562574, BD Biosciences), intracellular staining was performed in perm/wash buffer (BD Biosciences) at $4^{\circ} \mathrm{C}$ for 50 minutes. Data were acquired using a LSRFortessa flow cytometer and analyzed using FlowJo v10.4.0 software (Supplementary Fig. S1). Compensation controls were performed using splenocytes. All antibodies (clones, catalogue number) were purchased from BioLegend: 
FITC CD3 (17A2, 100204), Brilliant Violet 510 ${ }^{\text {TM }}$ CD8a (53-6.7, 100752), PE CD25 (PC61, 102008), Brilliant Violet 421 ${ }^{\mathrm{TM}}$ FOXP3 (MF-14, 126419), APC IFN-y (XMG1.2, 505810), PerCP/Cyanine5.5 CD45 (30-F11, 103132), Brilliant Violet 421 TM NK-1.1 (PK136, 108741), PE CD86 (GL-1, 105008), FITC CD11b (M1/70, 101206), APC F4/80 (BM8, 123116), PE/Cy7 CD206 (C068C2, 141719), APC CD62L (MEL-14, 104412), PE/Dazzle ${ }^{\text {TM }} 594$ CD44(IM7, 103056).

\section{Colony formation assay}

Cells (500-8000/well) were seeded in 6-well plates. Cells were incubated with/without ICG-001 (5 $\mu \mathrm{M})$ for 48 hours then replaced with fresh medium. Cells in the control group and ICG-001 group were subsequently administrated different doses of $\operatorname{IR}(0,2,4,6$, and $8 \mathrm{~Gy})$. Fourteen to twenty days after seeding, cells were fixed in methanol, and stained with crystal violet. The visible colonies (with $>50$ cells) were manually counted. The plating efficiency (PE) was calculated as the number colonies / the number of seeded cells $\times 100 \%$. The survival fraction is the colonies divided by the number of colonies corrected for the PE. All data were analyzed with GraphPad Prism 8 software, and survival curves were calculated using the single-hit multi-targeted model $\left(y=1-\left(1-\exp \left(-k^{\star} x\right)\right)^{\wedge} N\right)$.

\section{Immunoblotting}

Cells or tissues were lysed in RIPA buffer with protease inhibitors and phosphatase inhibitor. Cell extracts were separated on $12 \%$ SDS-PAGE gels and transferred to PVDF membranes (Millipore). Before incubated with specific primary antibody at $4{ }^{\circ} \mathrm{C}$ overnight, membranes were blocked with $5 \%$ skim milk in Trisbuffered saline/Tween 20 (TBST) for $1 \mathrm{~h}$ at room temperature. Subsequently, three washes were applied to the membranes, followed by secondary HRP-conjugated antibodies (1:5000) for 1 hour at room temperature. The immunoblots were detected using ECL (Thermo Fisher Scientific) according to the manufacturer's protocol. The following primary antibodies were used: anti- $\beta$-catenin (51067-2-AP, proteintech), anti-c-Jun(Ser139, 60A8, Cell Signaling Technology), anti-CyclinD1 (60186, proteintech), antiY-H2AX (Ser139, 20E3, Cell Signaling Technology), anti-cGAS (D3080, Cell Signaling Technology), antiSTING (D1V5L, Cell Signaling Technology), anti-p-STING(Ser365, D8F4W, Cell Signaling Technology), anti-IRF3 (D83B6, Cell Signaling Technology), anti-p-IRF3(Ser396, D601M, Cell Signaling Technology), anti-TBK1 (D1B4, Cell Signaling Technology), anti-p-TBK1(Ser172, D52C2, Cell Signaling Technology) and anti-GAPDH (Fdbio science, China).

\section{Immunofluorescence staining}

$5 \times 10^{4}$ HCC-LM3 or Hepa1-6 HCC cells were seeded on glass coverslips in a 24-well plate. Cells with/without ICG-001 treatment for 48 hours were administrated 6 Gy IR. After 0, 2, 8 hours of IR, cells were fixed with $4 \%$ paraformaldehyde in phosphate-buffered saline (PBS) for 15 minutes before permeabilized in $0.25 \%$ Triton X-100 in PBS containing 5\% BSA for 15 minutes. The permeabilized and blocked cells were incubated overnight at $4^{\circ} \mathrm{C}$ with primary antibodies against $\mathrm{Y}-\mathrm{H} 2 \mathrm{AX}$ (Ser139, 20E3, Cell Signaling Technology) at a 1:100 dilution. Secondary antibodies used were Cy3-conjugated goat anti- 
rabbit lgG (1:100) (Bioworld). Nuclei were counterstained with DAPI. Carl Zeiss LSM880 confocal microscope was used for fluorescence microscopy.

\section{Hematoxylin-Eosin staining and immunohistochemistry}

Formalin-fixed, paraffin-embedded murine tumor tissues sections were used for Hematoxylin \& Eosin (H\&E) staining and immunohistochemistry (IHC). The sections (5 $\mu \mathrm{m}$ thick) were de-paraffinized, rehydrated and subjected to heat-induced antigens retrieval in $10 \mathrm{mM}$ sodium citrate buffer $(\mathrm{pH} 6.0)$. After blocked with $5 \%$ goat serum (SL034, Solarbio Life Sciences) and Minute ${ }^{\mathrm{TM}}$ Peroxidase Suppressor (WA005, Invent biotechnology Inc.), sections were incubated with anti y-H2AX (Ser139, 20E3, Cell Signaling Technology) at $4^{\circ} \mathrm{C}$ overnight and subsequently applied with the secondary antibody for 1 hour at room temperature. Sections were developed with DAB (GK500705, Gene Tech) and counterstained with hematoxylin and eosin.

\section{Statistical analysis}

The comparisons between three or more groups were performed using one-way ANOVA, followed by Tukey's multiple comparison test. The comparison of mice subcutaneous tumor volume from four groups was analyzed by using mixed-effects model, followed by Tukey's multiple comparison test. Survival curves for different groups of mice were generated using the Kaplan-Meier method and analyzed by performing the log-rank Mantel-Cox test. $P<0.05$ was considered as statistical significance. All graphs were plotted using GraphPad Prism 8.

\section{Results}

\section{ICG-001 exerts stronger effect in increasing anti-tumor efficacy and enhancing survival of radiotherapy in immune-proficient than immune-deficient mice}

To determine the treatment efficacy of ICG-001 in combination with radiotherapy, the Hepa1-6 tumorbearing nude mice were treated with ICG-001, radiotherapy, or combination of radiotherapy and ICG-001 (Fig. 1a). we destinated the day when the treatments started as day 0. In vehicle group, ICG-001 group, and radiotherapy group, the designated tumor volume endpoint $\left(1000 \mathrm{~mm}^{3}\right)$ was reached at day 8,10 , and 14, respectively (Fig. 1b). While radiotherapy and ICG-001 combination group reached the endpoint on day 22. Compared with radiotherapy alone, combination treatment significantly inhibited tumor growth (Fig. $1 \mathrm{~b}$ and c; mean tumor volume \pm SEM at day 12: $946.00 \pm 54.00 \mathrm{~mm}^{3}$ radiotherapy vs. 653.00 $\pm 35.01 \mathrm{~mm}^{3}$ radiotherapy plus ICG-001, $P<0.0001$ ). In addition, combination therapy significantly prolonged survival compared with radiotherapy alone, which was shown by higher median survival time (Fig. 1 d; 10 days radiotherapy vs. 17 days radiotherapy plus ICG-001, $P<0.0001$ )

Interestingly, in Hepa1-6 tumor-bearing C57BL/6 mice, we found that radiotherapy alone and combination treatment group did not reach the endpoint yet until day 40. In addition, the combination of ICG-001 with radiotherapy almost eliminated the xenografts (Fig. 1e and f, mean tumor volume \pm SEM at day 40 : 
$787.75 \pm 186.88 \mathrm{~mm}^{3}$ radiotherapy vs. $32.27 \pm 32.27 \mathrm{~mm}^{3}$ radiotherapy plus ICG-001, $\left.P=0.0011\right)$. Furthermore, the median survival time of the mice treated with vehicle, ICG-001, and radiotherapy alone was 20,30 , and 40 days, respectively. Whereas the survival of the mice in combination group was $90 \%$ at the end of observation period (Fig. 1g). In addition, the xenografts from C57BL/ 6 mice showed higher complete response rate than those in nude mice after the combination therapy $(90 \% \mathrm{C} 57 \mathrm{BL} / 6$ mice vs. $0 \%$ nude mice). Therefore, although the addition of ICG-001 to radiotherapy significantly inhibited tumor growth and prolonged the survival time of tumor bearing mice in both immune-proficient C57BL/ 6 mice than immune-deficient nude mice, the synergistic effect was dramatically stronger with the presence of normal immune condition.

\section{Combination of ICG-001 and radiotherapy improves immune microenvironment in mice HCC xenografts}

Previous results have shown that ICG-001 displayed more substantial therapeutic effect with radiotherapy in the context of immune, we hypothesized that ICG-001 could regulate tumor immune microenvironment (TME) in combination with radiotherapy in HCC. To confirm this, we analyzed the alterations of varied tumor-infiltrating lymphocytes (TILs) in xenografts from Hepa1-6-bearing C57BL/6 mice treated with ICG-001, radiotherapy, or the combination. Compared with vehicle, radiotherapy evaluated the ratio of $\mathrm{TIL} \mathrm{CD8}^{+} / \mathrm{CD}^{+}{ }^{+}$T cells (Fig. $2 \mathrm{a}$; $39.31 \% \pm 6.00 \%$ vehicle vs. $51.54 \% \pm 5.57 \%$ radiotherapy, $P=0.025)$. Meanwhile, radiotherapy alone also increased the number of TIL CD $8^{+} \mathrm{T}$ cells, but the difference did not reach statistical significance (Fig. 2b; $42.85 \pm 28.93$ vehicle vs. $120.70 \pm 71.77$ radiotherapy, $P=0.6666$ ). Moreover, the addition of ICG-001 further increased the percentage (Fig. 2a; $51.54 \% \pm 5.57 \%$ radiotherapy vs. $67.62 \% \pm 7.91 \%$ radiotherapy plus ICG-001, $P=0.0021$ ) of TIL CD8 ${ }^{+} \mathrm{T}$ cells, and the number (Fig. 2 b; $120.70 \pm 71.77$ radiotherapy vs. $325.30 \pm 204.60$ radiotherapy plus ICG$001, P=0.0321)$.

Next, we explored the role of ICG-001 on effector function of TIL CD8 ${ }^{+}$and $C D 4^{+} T$ cells. After the stimulation of IFN- $\gamma$ production by phorbol 12-myristate 13-acetate (PMA) and ionomycin, we found that the percentage of TIL CD8 ${ }^{+}$T cells that produced IFN- $\gamma$ in combination treatment group was significantly higher than that in other treatment groups (Fig. 2c; $23.03 \% \pm 7.79 \%$ radiotherapy plus ICG-001 vs. $6.95 \% \pm$ $2.65 \%$ vehicle, $P<0.0001$; vs. $14.80 \% \pm 3.926 \%$ ICG-001, $P=0.0424$; vs. $12.48 \% \pm 3.87 \%$ radiotherapy, $P=$ 0.0073 ). We additionally examined the percentage of TIL CD ${ }^{+} \mathrm{T}$ cells that produced IFN- $\gamma$. Similar with $\mathrm{CD}^{+} \mathrm{T}$ cells, compared with other treatment regimens, the combination of ICG-001 and radiotherapy significantly increased the percentage of TIL IFN- $\gamma^{+} \mathrm{CD} 4^{+} \mathrm{T}$ cells (Fig. $2 \mathrm{~d} ; 34.13 \% \pm 14.93 \%$ radiotherapy plus ICG-001 vs. $6.76 \% \pm 4.87 \%$ vehicle, $P<0.0001$; vs. $13.08 \% \pm 4.82 \%$ ICG-001, $P=0.0018$; vs. $10.53 \% \pm$ $3.92 \%$ radiotherapy, $P=0.0005)$.

Besides the positive effect, radiation also confers negative effect on TME. In our study, the upregulated number of TIL Tregs was observed in radiotherapy alone group (Fig. 2e; $4.12 \pm 1.50$ vehicle vs. $6.25 \pm$ 1.49 radiotherapy, $P=0.0246$ ). The addition of ICG-001 to radiotherapy reduced the infiltration of Tregs (Fig. 2e; $6.25 \pm 1.49$ radiotherapy vs. $4.66 \pm 0.86$ radiotherapy plus ICG-001, $P=0.1225$ ), but the 
difference did not reach statistical significance. Meanwhile, compared with radiotherapy, combination treatment significantly increased the $\mathrm{CD} 8^{+} /$Tregs ratio (Fig. 2 f; $23.02 \% \pm 12.49 \%$ radiotherapy vs. $54.84 \%$ $\pm 16.88 \%$ radiotherapy plus ICG- $001, P=0.0398$ ).

We also investigated the status of innate immunity in xenografts from the mice with different treatments. No differences in the numbers of macrophage, dendritic cells, NK cells, and M2/macrophage ratio were observed between radiotherapy and combination treatment (Fig. 3a, b, c, and d, Supplementary Fig. S2). However, we found that adding ICG-001 to radiotherapy dramatically increased the ratio of M1/macrophage (Fig. 3 ; $44.00 \% \pm 6.99 \%$ radiotherapy vs. $57.55 \% \pm 7.11 \%$ radiotherapy plus ICG-001, $P$ $=0.0234)$.

In summary, the addition of ICG-001 to radiotherapy activated TME in mice HCC xenografts by increasing the frequency and function of cytotoxic TILs (CD8 ${ }^{+}$and $\mathrm{CD} 4^{+} \mathrm{T}$ cells), and decreasing the infiltration of suppressive TILs (Tregs).

\section{ICG-001 increases radiation-induced DNA damage of HCC cells in vitro and in vivo}

Previously we have shown that ICG-001 increased radiosensitivity in nude mice, which implied that other than immune-editing, ICG-001 also played a critical role in radio-sensitizing regulation. To confirm this, by performing clonogenic survival assays, we found that ICG-001 inhibited colony formation of both Hepa16 and HCC-LM3 cells (Fig. 4a and b). These findings indicated the role of ICG-001 in increasing instinct radiosensitivity of HCC cells. Since it is widely reported that radiation-induced DNA damage is closely associated with radiosensitivity. Thus, we investigated the effect of ICG-001 on radiation-induced doublestrand breaks (DSBs) by detecting the accumulation nuclear foci of $\mathrm{Y}-\mathrm{H} 2 \mathrm{AX}$ by immunofluorescence staining, a specific marker of DSBs, at a serial timepoints after IR treatment. We found that there were significantly more cells with > 10 foci in combination treatment group than radiation alone group (Fig. 4c and e). In addition, compared with radiotherapy, the addition of ICG-001 caused higher protein level of $\mathrm{Y}^{-}$ H2AX post IR (Fig. 4d and f). Consistent with in vitro results, we observed more cell death and higher level of $\mathrm{Y}-\mathrm{H} 2 \mathrm{AX}$ in mice treated with combination treatment than radiotherapy alone (Fig. $4 \mathrm{~g}$ and $\mathrm{h}$ ). Collectively, our results demonstrated that ICG-001 enhanced radiation-induced DNA damage and increased radiosensitivity of HCC cells in vitro and in vivo.

\section{ICG-001 promotes the anti-tumor efficacy and immune-improvement effect of radiation via activation of cGAS/STING}

Previous reports have demonstrated that radiation promoted T cell infiltration through producing doublestrand DNA (dsDNA) and activating cGAS/STING signaling pathway. In this study, we showed that the addition of ICG-001 to radiotherapy increased radiation-induced DNA damage, improved TME of HCC and prolonged HCC-bearing mice survival. Next, we investigated whether the anti-tumor and immune-editing role of ICG-001 in combination with radiotherapy relies on CGAS/STING signaling pathway activation. By analyzing the protein levels of several key factors involved in CGAS/STING signaling pathway, including cGAS, p-STING, and p-TBK1, in Hepa1-6 or HCC-LM3 cells treated with different treatment regimens. We 
found that ICG-001 further enhanced radiation-induced cGAS/STING signaling pathway activation in HCC cells (Fig. 5a, Supplementary Fig. S3). Consistent with these findings in vitro, similar results were observed in xenografts derived from the mice treated with radiotherapy or combination treatment (Fig. 5b). All these data implied that ICG-001 might exert an anti-tumor effect with radiotherapy by boosting the radiation-induced activation of CGAS/STING pathway in HCC, which shed light on the possibility that blockage of CGAS/STING pathway could weaken the synergistic therapeutic efficacy of ICG-001 with radiotherapy. By challenging the tumor-bearing mice with STING inhibitor C-176, we found that C-176 impaired the anti-tumor effect of the combination treatment (Fig. $5 \mathrm{c}$ and d; day 20: $499.00 \pm 158.98 \mathrm{~mm}^{3}$ combination therapy vs. $1246.90 \pm 393.74 \mathrm{~mm}^{3}$ combination therapy plus $\left.C-176, P<0.0001\right)$. Moreover, C-176 decreased the percentage (Fig. 5e and Supplementary Fig. S4a; combination therapy $56.83 \% \pm$ $11.92 \%$ vs. combination therapy plus $\mathrm{C}-17634.33 \% \pm 6.16 \%, P=0.0003$ ) and number of TIL CD $8^{+} \mathrm{T}$ cells (Fig. 5f; combination therapy $272.10 \pm 136.00$ vs. combination therapy plus C-176 $54.33 \pm 37.89, P=$ 0.0002), and the IFN-y production activity of CD8 $8^{+} \mathrm{T}$ cells (Fig. $5 \mathrm{~g}$ and Supplementary Fig. S4b; combination therapy $19.89 \% \pm 5.31 \%$ vs. combination therapy plus C- $1766.92 \% \pm 3.02 \%, P=0.0006$ ). Taken all these together, our findings indicated that ICG-001 exerted the synergistic tumor-control and TME-activation effect with radiotherapy in a cGAS/STING dependent manner.

\section{The addition of ICG-001 to radiotherapy prevented HCC recurrence}

We analyzed the memory status of $\mathrm{CD}^{+} \mathrm{T}$ cells in mice spleens. Compared with radiotherapy, the addition of ICG-001 increased the percentage of TIL CD8 ${ }^{+}$effector memory $\mathrm{T}\left(\mathrm{T}_{\mathrm{EM}}\right)$ cells (Fig 6a; radiotherapy $18.28 \% \pm 1.52 \%$ vs. radiotherapy plus ICG- $00139.23 \% \pm 6.03 \%, P<0.0001$ ). It is well demonstrated that the activation and memory status of $\mathrm{CD}^{+}$effector $\mathrm{T}$ cells is closely related with longterm tumor control, next we determined the effect of ICG-001 on tumor recurrence. We re-implanted new HCC tumors into the contralateral flank of the tumor bearing mice with complete course of varied treatments, and then monitored the new tumors in each group. Results revealed that, compared with radiotherapy alone, the addition of ICG-001 retarded the speed of tumor growth and reduced the secondary tumor volume, suggestive of the potent inhibitory role of ICG-001 with radiotherapy in secondary tumor growth (Fig. $6 \mathrm{~b}$ and c). These data suggested that combination treatment improved immunologic memory and delayed recurrence of HCC by increasing $\mathrm{T}_{\mathrm{EM}}$ cells in tumors.

\section{Discussion}

In our study, we found that the addition of ICG-001 enhanced the anti-tumor and survival-prolong efficacy of radiotherapy on the HCC cells-derived xenografts regardless of the immune state of the mice. Nevertheless, this synergetic effect was more significant in immune-proficient mice than immunedeficient mice, which suggested that other than tumor cell autonomous effect, ICG-001 also boosted the non-tumor cell autonomous effect of radiotherapy. By analyzing the tumor infiltrating lymphocytes, we found that, compared with radiation alone group, the total infiltration amount and the ones with IFN- $y$ secretion ability of $\mathrm{CD}^{+} \mathrm{T}$ cells increased, meanwhile the $\mathrm{CD} 8^{+} /$Tregs ratio evaluated in combination 
treatment. These findings uncovered that the addition of ICG-001 exerts not only radio-sensitizing but also a synergistic immune microenvironment stimulating effect upon radiotherapy.

Both of the stimulatory and inhibitory effects of radiation on immune microenvironment have been well reported[12, 24]. However, as for the Hepal-6 subcutaneous xenograft model in our study, we found that although there is a trend that radiation increased the number and the IFN- $\gamma$ secreting ability of TIL CD $8^{+}$T lymphocytes, and also decreased the number of Tregs and increased the $\mathrm{CD} 8^{+} /$Tregs ratio. But the difference did not reach statistical significance. Analogously, the similar trends regarding TIL CD $8^{+} \mathrm{T}$ cells and Tregs were observed in CT26 colorectal cells-derived xenografts upon radiotherapy reported by Vendetti et al[25]. While these differences reached significance at day 9 but not day 5 after radiotherapy. Taken this previous study into consideration, besides that we focused on distinct cancer types, the other possible explanation for the insignificant alterations of TILs in our case may be the timepoints we analyzed TILs at day 7 after radiotherapy.

The interaction and communication between radioresistance and TME of cancer cells is complicated[26, 27]. Basically, the instinct radioresistant property protects cancer cell form death, thus generating less dsDNA and weakening antigen presentation upon radiation[28]. As the result, the TME cannot be substantially activated, and the cytotoxic $T$ cells including TIL CD8 ${ }^{+} \mathrm{T}$ cells cannot be sufficiently primed[18]. In return, numerous studies have reported that $\mathrm{CD} 8^{+} \mathrm{T}$ cell infiltration after radiation is critical for tumor response to radiotherapy, deletion of $\mathrm{CD} 8^{+} \mathrm{T}$ cell caused radioresistance in cancer cells[12, 13]. Thus, one key to efficiently improve the efficacy of radiotherapy is to target the regulators involved in both cell instinct radioresistance and TME suppression. Hou et al. has identified that NF-KB pathway, which plays a crucial role in radioresistance in tumor cells, also participates in radiation-induced anti-tumor immunity[29]. Moreover, by inhibiting non-canonical NF-kB pathway, DCs were activated and the therapeutic efficiency of radiotherapy was enhanced in cancer cells[29]. These findings suggested that targeting non-canonical NF-KB may be designed as a therapeutic strategy that benefits both cell intrinsic radiosensitivity and TME upon radiation.

As for HCC, there are about 30-40\% HCC patients carrying wnt/ $\beta$-catenin activation mutation[14, 20,30]. $W n t / \beta$-catenin signaling was reported to induce radioresistance in several human cancers including head and neck, breast, nasopharyngeal, esophageal, glioblastoma, and colorectal cancers[31-36]. In addition, wnt/ $\beta$-catenin signaling was associated with immune-desert TME[37-39]. For example, Sia et al. demonstrated that HCC samples with wnt/ $\beta$-catenin activation showed significantly lower enrichment score for several immune signatures, especially T cells[40]. A preclinical study also demonstrated that $\beta$ catenin activation led to a defective recruitment of DCs and antigen-specific T cells[18]. As a following consequence, anti-tumor immune response was impaired. Taken all these reports into consideration, wnt/ $\beta$-catenin seems to be a promising signaling pathway in coordinating cancer cells and TME to protect HCC cells from radiation-induced damage. In our study, we found that ICG-001 increased radiosensitivity of HCC cells by enhancing radiation-induced DNA damage, and meanwhile augmented cell-killing T cells infiltration by strengthening radiation-induced cGAS/STING signaling. Thus, targeting wnt/ $\beta$-catenin 
might be an appropriate approach for increasing radiosensitivity and activating TME, finally promoting anti-tumor efficacy of radiotherapy and creating suitable condition for radioimmunotherapy in a large group of HCC patients who carries wnt/ $\beta$-catenin alterations.

It is widely demonstrated that DCs are critical in priming $C D 8^{+} \mathrm{T}$ cells, and $\beta$-catenin activation downregulated several chemokines required for DCs recruitment, thus impairing anti-tumor immunity[41]. According to this report, inhibition of $\beta$-catenin should increase the infiltrating DCs in HCC. However, in our study, ICG-001 did not significantly altered the numbers of DCs in xenografts. The reason for this contradiction was unclear, but we can get a clue form the observation in one study showing that radiation-induced dsDNA translocated from tumor cells to DCs and activated cGAS/STING signaling, and then promoted the antigen processing and presenting capacities of DCs[29]. Our results revealed that ICG001 further evaluated radiation-induced cGAS/STING activation and increased the percentage of TIL $\mathrm{CD}^{+} \mathrm{T}$ cells with IFN- $\gamma$ production ability. We assumed that ICG-001 might exert stronger effect in potentiating the function rather than priming the infiltration of DCs via enhancing radiosensitivity and stimulating CGAS/STING signaling pathway of HCC cells in our context.

Interestingly, we found that compared with non-treated group, the $\mathrm{CD} 8^{+} / \mathrm{CD} 3^{+}$ratio in xenografts from tumor-bearing mice treated with ICG-001 was dramatically increased. However, the addition of radiotherapy with ICG-001 did not further increase this ratio, even though the treatment efficacy was remarkably better in combination group than ICG-001 alone group. Given the evidence establishing radiotherapy led to further increase in the percentage of TIL IFN $-\gamma^{+} \mathrm{CD} 8^{+}$and IFN- $\gamma^{+} \mathrm{CD} 4^{+} \mathrm{T}$ cells upon ICG-001 treatment, we speculated that, compared with ICG-001, the additional treatment benefit of the combination therapy was mainly dependent on potentiating the cytotoxic ability of $\mathrm{CD}^{+} \mathrm{T}$ cells but not increasing the absolute frequency of infiltrating $\mathrm{CD} 8^{+} \mathrm{T}$ cells.

Radiation-generated dsDNA plays a crucial role activating cGAS/STING signaling pathway in both tumor cells and immune cells $[9,42,43]$. For example, radiation-activated cGAS/STING mediated tumorsuppressive effects, meanwhile, triggers the capability of DCs and promotes the anti-tumor immunity[44]. In our study, we found that the combination of ICG-001 with radiotherapy led to the activation of cGAS/STING pathway and increased TIL IFN $-\gamma^{+} \mathrm{CD}^{+} \mathrm{T}$ cells infiltrates in HCC tissues. These observations shed light on the possibility that the blockage of cGAS/STING signaling pathway could weaken the anti-tumor and immune-stimulatory effect of ICG-001. As our data shown, the addition of STING inhibitor $\mathrm{C}-176$ reduced the percentage of TIL IFN- $\gamma^{+} \mathrm{CD}^{+} \mathrm{T}$ cells in xenografts from HCC tumorbearing mice treated with the combination of radiotherapy and ICG-001. Moreover, C-176 impaired the anti-tumor efficacy of combination therapy in tumor-bearing mice.

As a potent antagonist of CBP/ $\beta$-catenin-mediated transcription, ICG-001 displayed cytotoxic effect in varied types of cancers. For example, ICG-001 induced apoptosis against T-cell acute lymphoblastic leukemia cells in combination with ZSTK-474, a PI3K inhibitor [22]. ICG-001 has also shown to increase sensitivity to cisplatin in platinum-resistant ovarian cancer cells[45]. Importantly, numerous clinical studies are being conducted to explore the effect of wnt/ $\beta$-catenin inhibitors in varied types of tumors, 
including pancreatic adenocarcinoma, colorectal cancers, and acute myeloid leukemia[46-48]. As the second-generation clinical-grade wnt/ $\beta$-catenin antagonist, PRI-724 has already entered early stage of clinical trials for advanced solid tumors (NCT01302405), and advanced myeloid malignancies (NCT01606579). Moreover, PRI-724 was well-tolerated by patients with HCV cirrhosis[23]. Here, we revealed that ICG-001 dramatically enhanced radiosensitivity, meanwhile promoted radiation-induced TME activation and boosted immunologic memory in HCC tumor-bearing mice. Thus, ICG-001 in combination with radiotherapy prolonged survival, and delayed recurrence of the tumor-bearing mice. Although the safety evidence is still lacking in cancer patients, our data provide a promising cure role of ICG-001 to combine with either radiotherapy or immunoradiotherapy.

Zhang et al. reported that ICG-001 decreased sphere formation and the migration capacity of glioma stem-like cells[49]. In addition, Cui et al. has shown that ICG-001 effectively inhibited the metastatic progression of HCC tumors in both cell line and patient-derived xenograft mouse models[50]. Moreover, PRI-724, the derivative of ICG-001, was reported to induce CD8 ${ }^{+}$T-cells infiltrates and IFN- $\gamma$ production in liver metastatic lesions in a mouse model of colon cancer with liver metastasis[51]. The combination treatment of anti-PD-L1 antibody and PRI-724 resulted in regression of tumor growth. Analogously, we found that ICG-001 also increased IFN- $\gamma$ production ability of $C D 8^{+} T$ cells in our context, which implied that combining ICG-001 and radioimmunotherapy may be a promising therapeutic approach to eradicate the in situ and the metastatic cancer cells in HCC patients.

In this study, we showed the two-sided benefits of ICG-001 combined with radiotherapy on both radiosensitizing and immune-editing in HCC. Our data raised the possibility that ICG-001 might be applicable for the combination therapy with radiotherapy or radioimmunotherapy in HCC patients. Our study was of high clinical significance, and the combination treatment paradigm merits evaluation on future clinical trials in HCC patients.

\section{Conclusion}

In summary, we have demonstrated that the addition of ICG-001 to radiotherapy displayed stronger synergistic anti-tumor efficacy and survival extension in immune-proficient mice than in immune-deficient mice. Compared with radiotherapy alone, the TME of the xenografts from the mice treated with the combination of ICG-001 and radiotherapy was improved, exhibited by the increased infiltration and IFN-Y production ability of TIL CD8 ${ }^{+}$T cells, and decreased number of TIL Tregs. In addition, ICG-001 increased DNA damage response and radiosensitivity of HCC cells. As the consequence, ICG-001 further enhanced the activation of radiation-induced CGAS/STING signaling pathway, and STING inhibitor C-176 impaired the anti-tumor efficacy of combination therapy. Importantly, ICG-001 also resulted in an increase in the percentage of splenic $\mathrm{CD} 8^{+} \mathrm{T}_{\mathrm{EM}}$, thus preventing recurrence in tumor-bearing mice.

Taken all these two-sided benefits of ICG-001 combined with radiotherapy on both HCC intrinsic radiosensitivity and TME together, our data suggested a novel potential synergistic therapeutic approach 
to combine with radiotherapy and radioimmunotherapy in HCC patients. Remarkedly, this study provided a preclinical evidence for future clinical human studies into the treatment of HCC.

\section{Abbreviations}

HCC: hepatocellular carcinoma; DC: dendritic cell; DDR: DNA damage repair; dsDNA: double-strand DNA; IR: ionizing radiation; SBRT: stereotactic body radiation therapy; TME: tumor immune microenvironment; TIL: tumor-infiltrating lymphocyte; Tregs: regulatory $T$ cells; $T_{E M}$ : effector memory $T$ cell; IFN- $\gamma$ : interferon gamma; PMA: phorbol 12-myristate 13-acetate.

\section{Declarations}

\section{Ethics approval and consent to participate}

All experimental procedures were approved by Southern Medical University Animal Management and Use Committee, and performed in accordance with all applicable international, national, and institutional guidelines for the care and use of animals.

\section{Consent for publication}

Not applicable.

\section{Availability of data and materials}

The data generated and/or analyzed during the current study are available upon reasonable request to the corresponding author.

\section{Competing interests}

The authors declare that they have no competing interest.

\section{Funding}

This work was supported by the National Nature Science Foundation of China (Grant Nos. 81903133 and 81702390), Chinese Postdoctoral Science Foundation (2020M672736), Natural Science Foundation of Guangdong Province (Grant No. 2017A030310105) and Guangzhou Technology Project (No.201906010087).

\section{Authors' contributions}

Jingyuan Sun, Chuanhui Cao designed and guided the research. Yan Huang, Hailong Sheng and Yazhi Xiao performed most of the experiments. Zhenru Zhu and Yiyao Chen wrote the manuscript. Zhihong Zhang analyzed the data. All authors reviewed and approved the final manuscript. 
Acknowledgements

Not applicable.

\section{References}

1. Bray F, Ferlay J, Soerjomataram I, Siegel R L, Torre L A, Jemal A, Global cancer statistics 2018 : GLOBOCAN estimates of incidence and mortality worldwide for 36 cancers in 185 countries. CA: a cancer journal for clinicians68, 394-424 (2018).

2. Benson A B, D'Angelica M I, Abbott D E, Abrams T A, Alberts S R, Anaya D A et al., Guidelines Insights: Hepatobiliary Cancers, Version 2.2019. Journal of the National Comprehensive Cancer Network: JNCCN17, 302-310 (2019).

3. Wahl D R, Stenmark M H, Tao Y, Pollom E L, Caoili E M, Lawrence T S et al., Outcomes After Stereotactic Body Radiotherapy or Radiofrequency Ablation for Hepatocellular Carcinoma. J Clin Onco/34, 452-459 (2016).

4. Bujold A, Massey C A, Kim J J, Brierley J, Cho C, Wong R K et al., Sequential phase I and II trials of stereotactic body radiotherapy for locally advanced hepatocellular carcinoma. J Clin Onco/31, 16311639 (2013).

5. Curtin $\mathrm{N} \mathrm{J}$, DNA repair dysregulation from cancer driver to therapeutic target. Nature reviews. Cancer12, 801-817 (2012).

6. Lord C J, Ashworth A, The DNA damage response and cancer therapy. Nature481, 287-294 (2012).

7. Barker $\mathrm{H}$ E, Paget $\mathrm{J}$ T, Khan A A, Harrington $\mathrm{K} J$, The tumour microenvironment after radiotherapy: mechanisms of resistance and recurrence. Nature reviews. Cancer15, 409-425 (2015).

8. Sheng H, Huang Y, Xiao Y, Zhu Z, Shen M, Zhou P et al., ATR inhibitor AZD6738 enhances the antitumor activity of radiotherapy and immune checkpoint inhibitors by potentiating the tumor immune microenvironment in hepatocellular carcinoma. Journal for immunotherapy of cancer8 (2020).

9. Yum S, Li M, Chen Z J, Old dogs, new trick: classic cancer therapies activate cGAS. Cell research30, 639-648 (2020).

10. Larson S M, Carrasquillo J A, Cheung N K, Press O W, Radioimmunotherapy of human tumours. Nature reviews. Cancer15, 347-360 (2015).

11. Kim K J, Kim J H, Lee S J, Lee E J, Shin E C, Seong J, Radiation improves antitumor effect of immune checkpoint inhibitor in murine hepatocellular carcinoma model. Oncotarget8, 41242-41255 (2017).

12. Lee $Y$, Auh S L, Wang Y, Burnette B, Wang Y, Meng Y et al., Therapeutic effects of ablative radiation on local tumor require CD8+ T cells: changing strategies for cancer treatment. Blood114, 589-595 (2009).

13. Chen H Y, Xu L, Li L F, Liu X X, Gao J X, Bai Y R, Inhibiting the CD8(+) T cell infiltration in the tumor microenvironment after radiotherapy is an important mechanism of radioresistance. Scientific 
reports8, 11934 (2018).

14. Khalaf A M, Fuentes D, Morshid A I, Burke M R, Kaseb A O, Hassan M et al., Role of Wnt/beta-catenin signaling in hepatocellular carcinoma, pathogenesis, and clinical significance. Journal of hepatocellular carcinoma5, 61-73 (2018).

15. Pez F, Lopez A, Kim M, Wands J R, Caron de Fromentel C, Merle P, Wnt signaling and hepatocarcinogenesis: molecular targets for the development of innovative anticancer drugs. Journal of hepatology59, 1107-1117 (2013).

16. Jun S, Jung Y S, Suh H N, Wang W, Kim M J, Oh Y S et al., LIG4 mediates Wnt signalling-induced radioresistance. Nature communications7, 10994 (2016).

17. Zhao Y, Tao L, Yi J, Song H, Chen L, The Role of Canonical Wnt Signaling in Regulating Radioresistance. Cell Physiol Biochem48, 419-432 (2018).

18. Ruiz de Galarreta M, Bresnahan E, Molina-Sanchez P, Lindblad K E, Maier B, Sia D et al., beta-Catenin Activation Promotes Immune Escape and Resistance to Anti-PD-1 Therapy in Hepatocellular Carcinoma. Cancer discovery9, 1124-1141 (2019).

19. Berraondo P, Ochoa M C, Olivera I, Melero I, Immune Desertic Landscapes in Hepatocellular Carcinoma Shaped by beta-Catenin Activation. Cancer discovery9, 1003-1005 (2019).

20. Harding J J, Nandakumar S, Armenia J, Khalil D N, Albano M, Ly M et al., Prospective Genotyping of Hepatocellular Carcinoma: Clinical Implications of Next-Generation Sequencing for Matching Patients to Targeted and Immune Therapies. Clinical cancer research : an official journal of the American Association for Cancer Research25, 2116-2126 (2019).

21. De Sousa E M F, Medema J P, Axing Wnt signals. Cell research22, 9-11 (2012).

22. Evangelisti C, Chiarini F, Cappellini A, Paganelli F, Fini M, Santi S et al., Targeting Wnt/beta-catenin and PI3K/Akt/mTOR pathways in T-cell acute lymphoblastic leukemia. J Cell Physio/235, 5413-5428 (2020).

23. Kimura K, Ikoma A, Shibakawa M, Shimoda S, Harada K, Saio M et al., Safety, Tolerability, and Preliminary Efficacy of the Anti-Fibrotic Small Molecule PRI-724, a CBP/beta-Catenin Inhibitor, in Patients with Hepatitis C Virus-related Cirrhosis: A Single-Center, Open-Label, Dose Escalation Phase 1 Trial. EBioMedicine23, 79-87 (2017).

24. Sanmamed M F, Chen L, A Paradigm Shift in Cancer Immunotherapy: From Enhancement to Normalization. Cel/175, 313-326 (2018).

25. Vendetti F P, Karukonda P, Clump D A, Teo T, Lalonde R, Nugent $K$ et al., ATR kinase inhibitor AZD6738 potentiates CD8+ T cell-dependent antitumor activity following radiation. The Journal of clinical investigation128, 3926-3940 (2018).

26. Boustani J, Grapin M, Laurent P A, Apetoh L, Mirjolet C, The 6th R of Radiobiology: Reactivation of Anti-Tumor Immune Response. Cancers11 (2019).

27. Sato H, Okonogi N, Nakano T, Rationale of combination of anti-PD-1/PD-L1 antibody therapy and radiotherapy for cancer treatment. Int J Clin Onco/25, 801-809 (2020). 
28. Reits E A, Hodge J W, Herberts C A, Groothuis T A, Chakraborty M, Wansley E K et al., Radiation modulates the peptide repertoire, enhances $\mathrm{MHC}$ class I expression, and induces successful antitumor immunotherapy. The Journal of experimental medicine203, 1259-1271 (2006).

29. Hou Y, Liang H, Rao E, Zheng W, Huang X, Deng L et al., Non-canonical NF-kappaB Antagonizes STING Sensor-Mediated DNA Sensing in Radiotherapy. Immunity49, 490-503 e494 (2018).

30. Cancer Genome Atlas Research Network. Electronic address w b e, Cancer Genome Atlas Research N, Comprehensive and Integrative Genomic Characterization of Hepatocellular Carcinoma. Cel/169, 1327-1341 e1323 (2017).

31. Alamoud K A, Kukuruzinska M A, Emerging Insights into Wnt/beta-catenin Signaling in Head and Neck Cancer. J Dent Res97, 665-673 (2018).

32. Fatima I, El-Ayachi I, Taotao L, Lillo M A, Krutilina R I, Seagroves T N et al., The natural compound Jatrophone interferes with Wnt/beta-catenin signaling and inhibits proliferation and EMT in human triple-negative breast cancer. PloS one12, e0189864 (2017).

33. Wang W, Wen Q, Luo J, Chu S, Chen L, Xu L et al., Suppression Of beta-catenin Nuclear Translocation By CGP57380 Decelerates Poor Progression And Potentiates Radiation-Induced Apoptosis in Nasopharyngeal Carcinoma. Theranostics7, 2134-2149 (2017).

34. Saito T, Mitomi H, Imamhasan A, Hayashi T, Mitani K, Takahashi M et al., Downregulation of sFRP-2 by epigenetic silencing activates the beta-catenin/Wnt signaling pathway in esophageal basaloid squamous cell carcinoma. Virchows Arch464, 135-143 (2014).

35. Major M B, Camp N D, Berndt J D, Yi X, Goldenberg S J, Hubbert C et al., Wilms tumor suppressor WTX negatively regulates WNT/beta-catenin signaling. Science (New York, N. Y.)316, 1043-1046 (2007).

36. Tenbaum S P, Ordonez-Moran P, Puig I, Chicote I, Arques O, Landolfi S et al., beta-catenin confers resistance to $\mathrm{PI3K}$ and $\mathrm{AKT}$ inhibitors and subverts FOXO3a to promote metastasis in colon cancer. Nature medicine18, 892-901 (2012).

37. Spranger S, Bao R, Gajewski T F, Melanoma-intrinsic beta-catenin signalling prevents anti-tumour immunity. Nature523, 231-235 (2015).

38. Xue J, Yu X, Xue L, Ge X, Zhao W, Peng W, Intrinsic beta-catenin signaling suppresses CD8(+) T-cell infiltration in colorectal cancer. Biomed Pharmacother115, 108921 (2019).

39. Hu-Lieskovan S, Homet Moreno B, Ribas A, Excluding T Cells: Is beta-Catenin the Full Story? Cancer cell27, 749-750 (2015).

40. Moeini A, Torrecilla S, Tovar V, Montironi C, Andreu-Oller C, Peix J et al., An Immune Gene Expression Signature Associated With Development of Human Hepatocellular Carcinoma Identifies Mice That Respond to Chemopreventive Agents. Gastroenterology157, 1383-1397 e1311 (2019).

41. Manicassamy S, Reizis B, Ravindran R, Nakaya H, Salazar-Gonzalez R M, Wang Y C et al., Activation of beta-catenin in dendritic cells regulates immunity versus tolerance in the intestine. Science (New York, N.Y.)329, 849-853 (2010). 
42. Marill J, Mohamed Anesary N, Paris S, DNA damage enhancement by radiotherapy-activated hafnium oxide nanoparticles improves cGAS-STING pathway activation in human colorectal cancer cells. Radiotherapy and oncology : journal of the European Society for Therapeutic Radiology and Oncology141, 262-266 (2019).

43. Barber G N, STING: infection, inflammation and cancer. Nature reviews. Immunology15, 760-770 (2015).

44. Sprooten J, Agostinis P, Garg A D, Type I interferons and dendritic cells in cancer immunotherapy. Int Rev Cell Mol Bio/348, 217-262 (2019).

45. Wu G, Cao L, Zhu J, Tan Z, Tang M, Li Z et al., Loss of RBMS3 Confers Platinum Resistance in Epithelial Ovarian Cancer via Activation of miR-126-5p/beta-catenin/CBP signaling. Clinical cancer research : an official journal of the American Association for Cancer Research25, 1022-1035 (2019).

46. Jiang X, Hao H X, Growney J D, Woolfenden S, Bottiglio C, Ng N et al., Inactivating mutations of RNF43 confer Wnt dependency in pancreatic ductal adenocarcinoma. Proceedings of the National Academy of Sciences of the United States of America110, 12649-12654 (2013).

47. Song S, Christova T, Perusini S, Alizadeh S, Bao R Y, Miller B W et al., Wnt inhibitor screen reveals iron dependence of beta-catenin signaling in cancers. Cancer research71, 7628-7639 (2011).

48. Bahrami A, Amerizadeh F, ShahidSales S, Khazaei M, Ghayour-Mobarhan M, Sadeghnia H R et al., Therapeutic Potential of Targeting Wnt/beta-Catenin Pathway in Treatment of Colorectal Cancer: Rational and Progress. J Cell Biochem118, 1979-1983 (2017).

49. Zhang Y, Wen Y L, Ma J W, Ye J C, Wang X, Huang J X et al., Tetrandrine inhibits glioma stem-like cells by repressing beta-catenin expression. Int J Onco/50, 101-110 (2017).

50. Cui Y, Wu X, Lin C, Zhang X, Ye L, Ren L et al., AKIP1 promotes early recurrence of hepatocellular carcinoma through activating the Wnt/beta-catenin/CBP signaling pathway. Oncogene38, 5516-5529 (2019).

51. Osawa Y, Kojika E, Nishikawa K, Kimura M, Osakaya S, Miyauchi H et al., Programmed cell death ligand 1 (PD-L1) blockade attenuates metastatic colon cancer growth in CAMP-response elementbinding protein (CREB)-binding protein (CBP)/beta-catenin inhibitor-treated livers. Oncotarget10, 3013-3026 (2019).

\section{Figures}


a

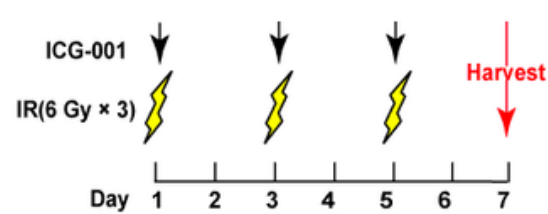

b

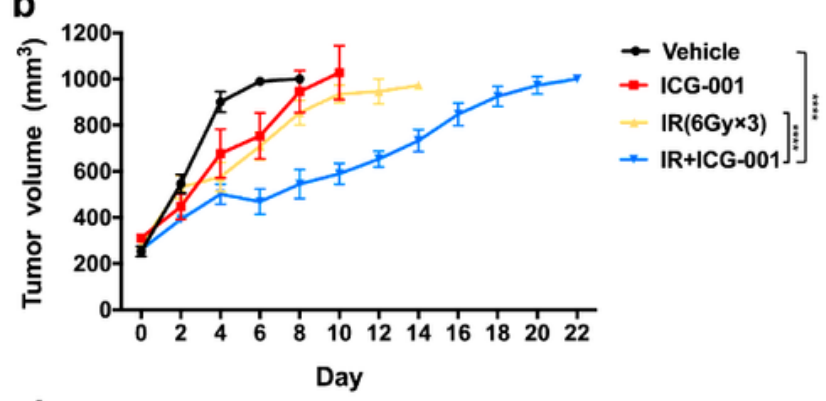

d

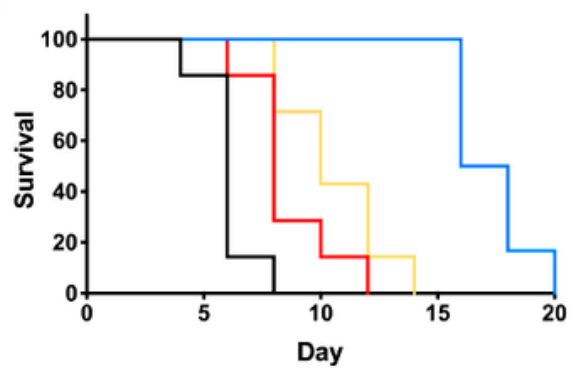

e

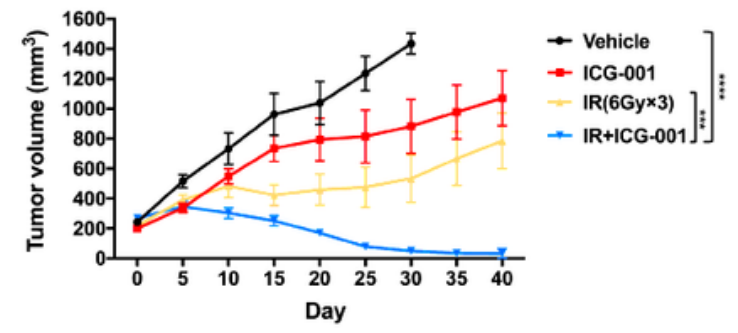

g

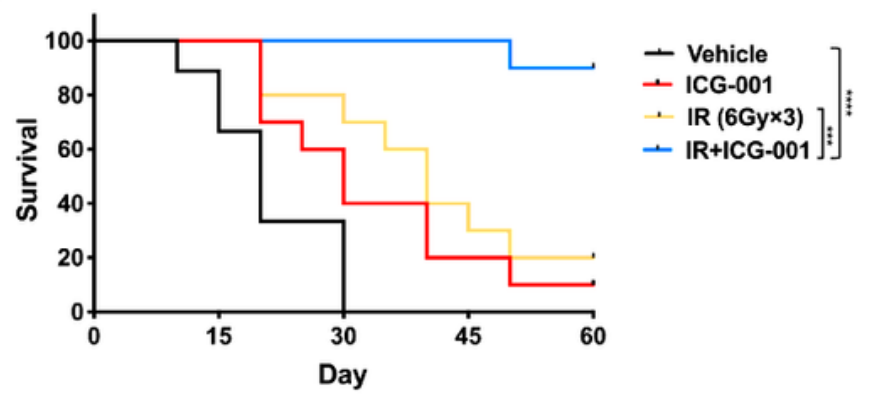

c

- Vehicle

- ICG-001

- IR (6Gy×3) ]:

- IR+ICG-001 $]$ ]

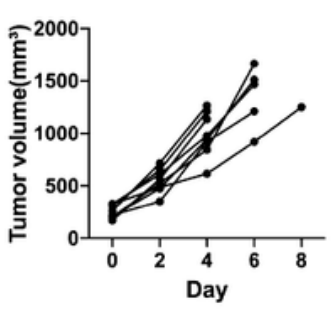

IR (6Gy×3)

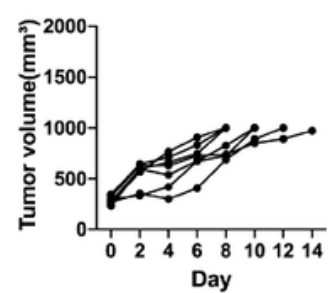

f

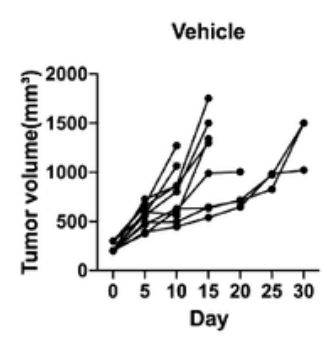

IR (6Gy×3)

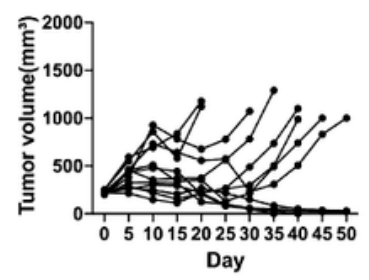

ICG-001

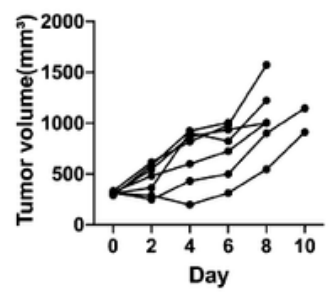

IR+ICG-001

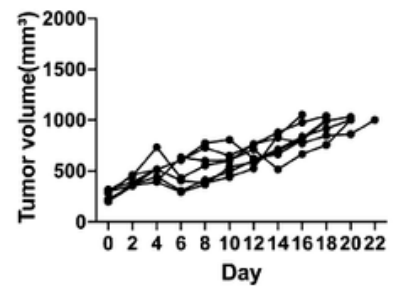

ICG-001

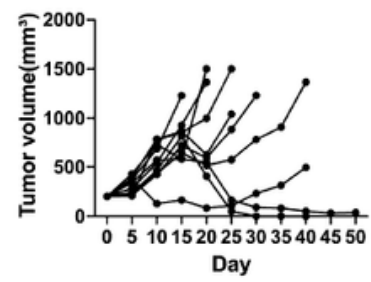

IR+|CG-001

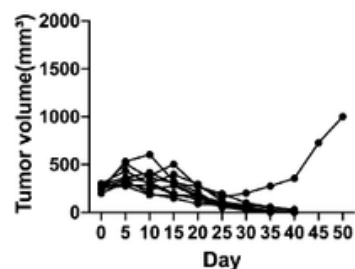

Fig.1

\section{Figure 1}

The addition ICG-001 exhibited stronger synergistic effect with radiotherapy in C57BL/6 mice than nude mice. (a) Treatment schedules of IR and ICG-001. (b-d) The tumor growth curves (b), the individual tumor volume curves (c), and Kaplan-Meier survival plots (d) of the nude mice receiving vehicle, IR alone, ICG001 alone or combined treatment. Data represent the mean \pm SEM. (e-g) The tumor growth curves (e), the individual tumor volume curves (f), and Kaplan-Meier survival plots (g) of the C57BL/6 mice receiving 
vehicle, IR alone, ICG-001 alone or combined treatment. Data represent the mean \pm SEM. For (b) and (e), statistical analyses were performed using a mixed-effects model, followed by Tukey's multiple comparison test. For (d) and (g), survival data was compared using the log-rank Mantel-Cox test. *** $\mathrm{P}<$ $0.001, * \star \star * \mathrm{P}<0.0001$.

a

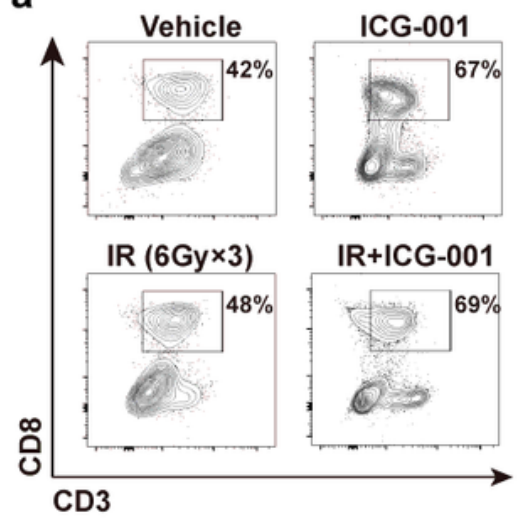

- Vehicle o IR

- ICG-001 - IR+ICG-001

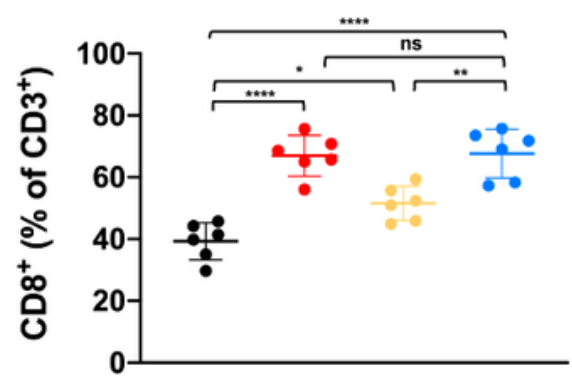

b

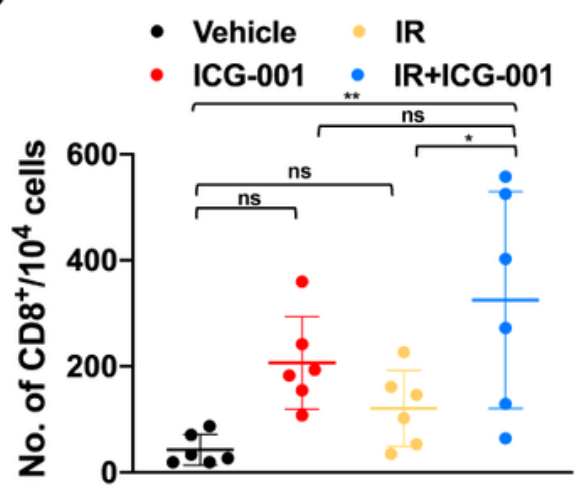

C
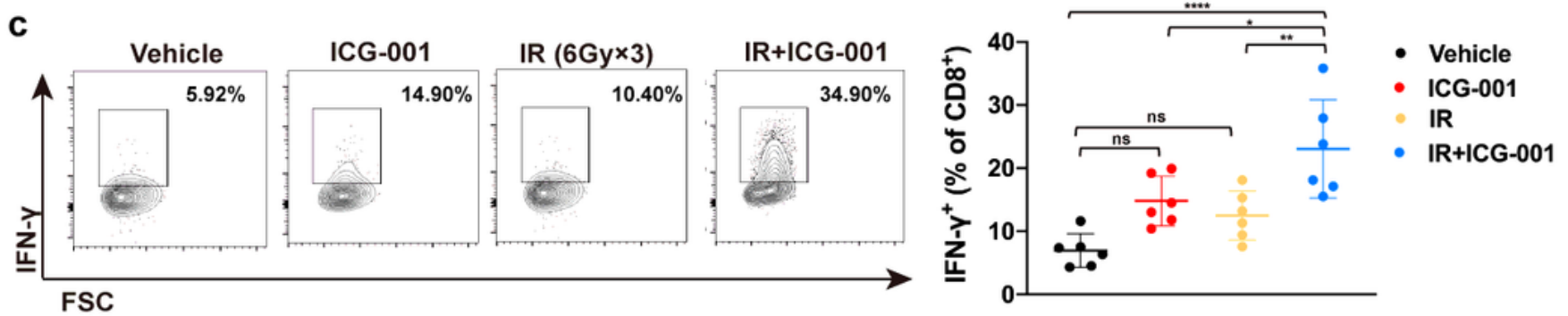

d
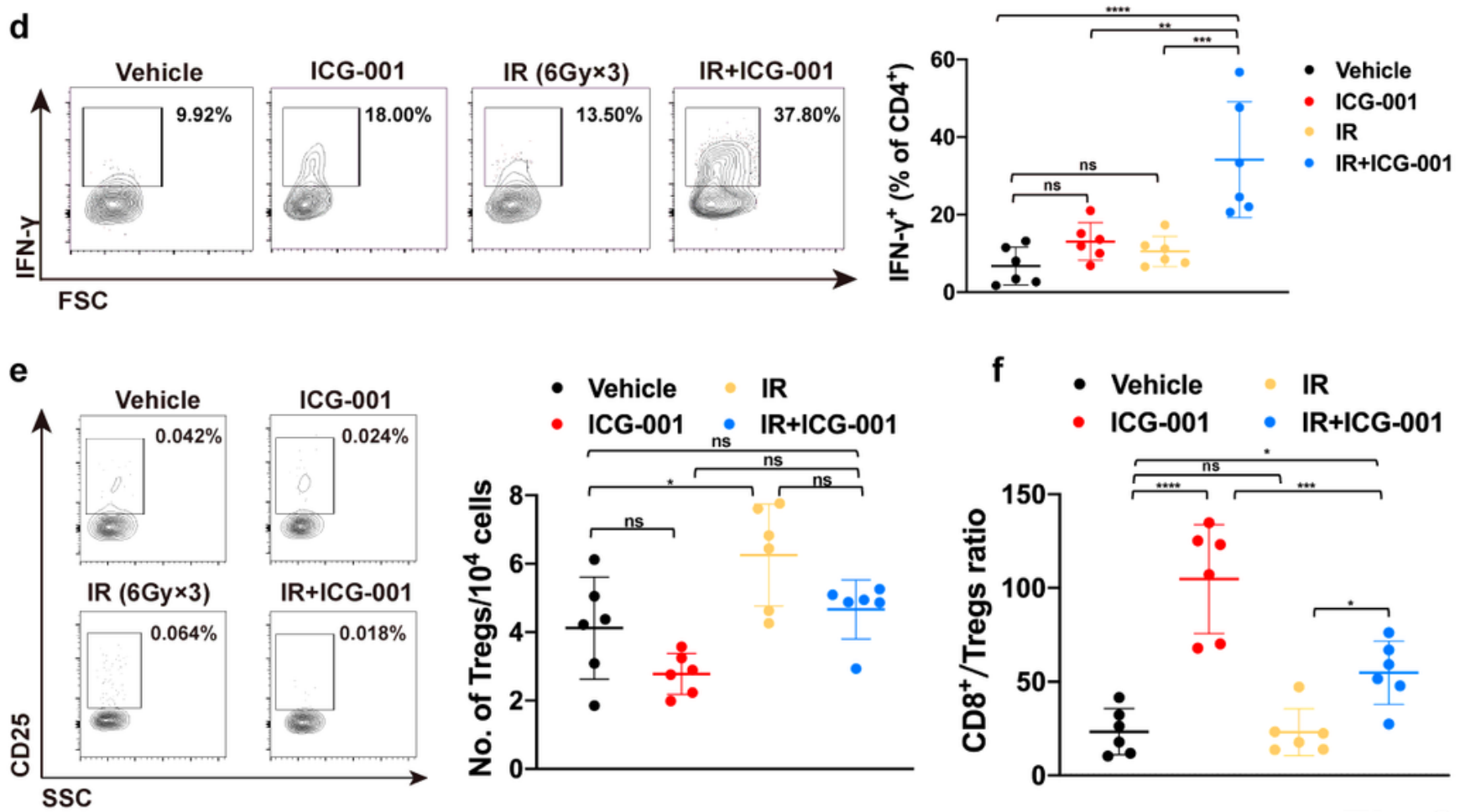

Fig.2

Figure 2 
ICG-001 increases radiation-induced infiltration and cytotoxic function of T cells in tumor microenvironment. (a) Representative contour plots (left panel) and quantification (middle panel) of CD8+/CD3+ ratios. (b) The quantitation of the number of TIL CD8+ T cells per 104 cells was shown. (c) Representative contour plots (left panel) and quantitation (right panel) of percentage of IFN- $\gamma$-secreting CD8+ TILs with PMA/ionomycin stimulation. (d) Representative contour plots (left panel) and quantitation (right panel) of percentage of IFN-Y-secreting CD4+ TILs (g) with PMA/ionomycin stimulation. (e) Representative contour plots (left panel) and quantitation (middle panel) of the percentage of Tregs (CD25+ Foxp3+) was shown. (f) The quantitation of the number of TIL Tregs per 104 cells was shown. Data represent the mean \pm SD. Ns, not significant, ${ }^{2} P<0.05,{ }^{*} \mathrm{P}<0.01,{ }^{\star \star \star} \mathrm{P}<0.001$, $\star \star \star * P<0.0001$ via ANOVA using Tukey's multiple comparisons test. 

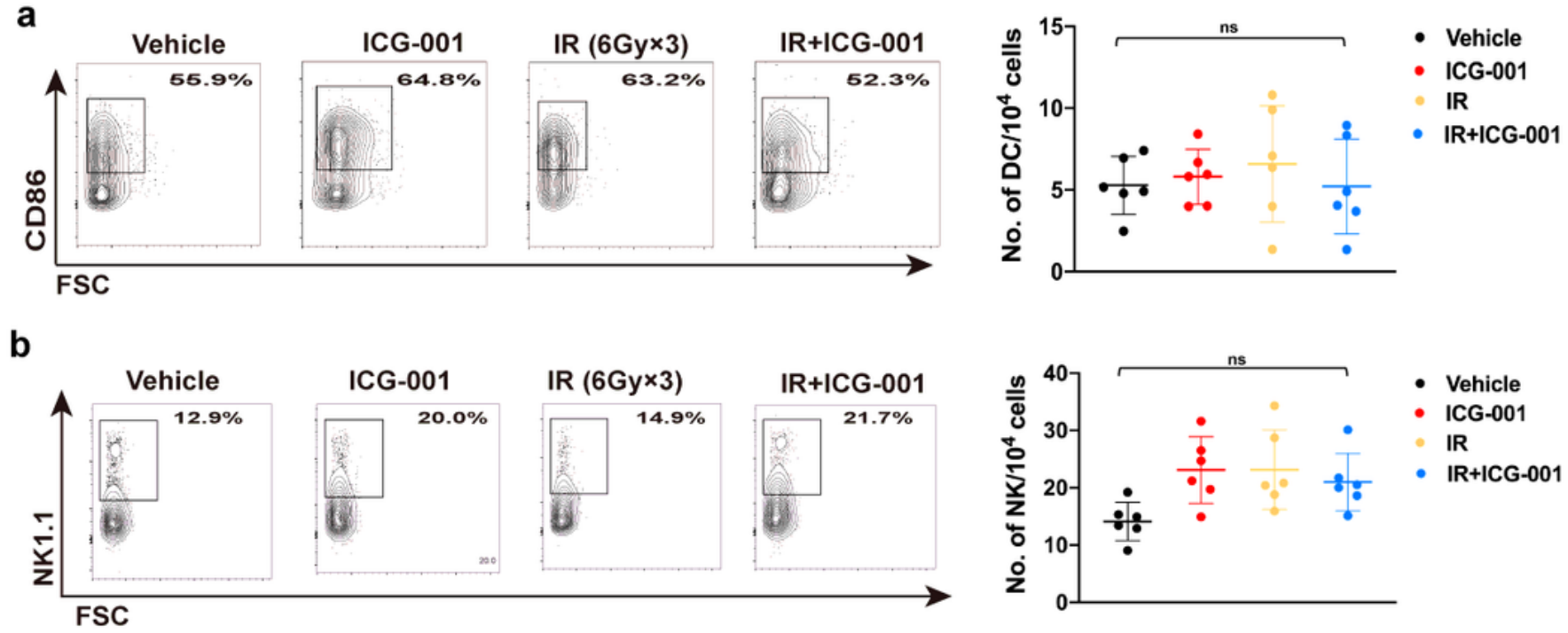

C
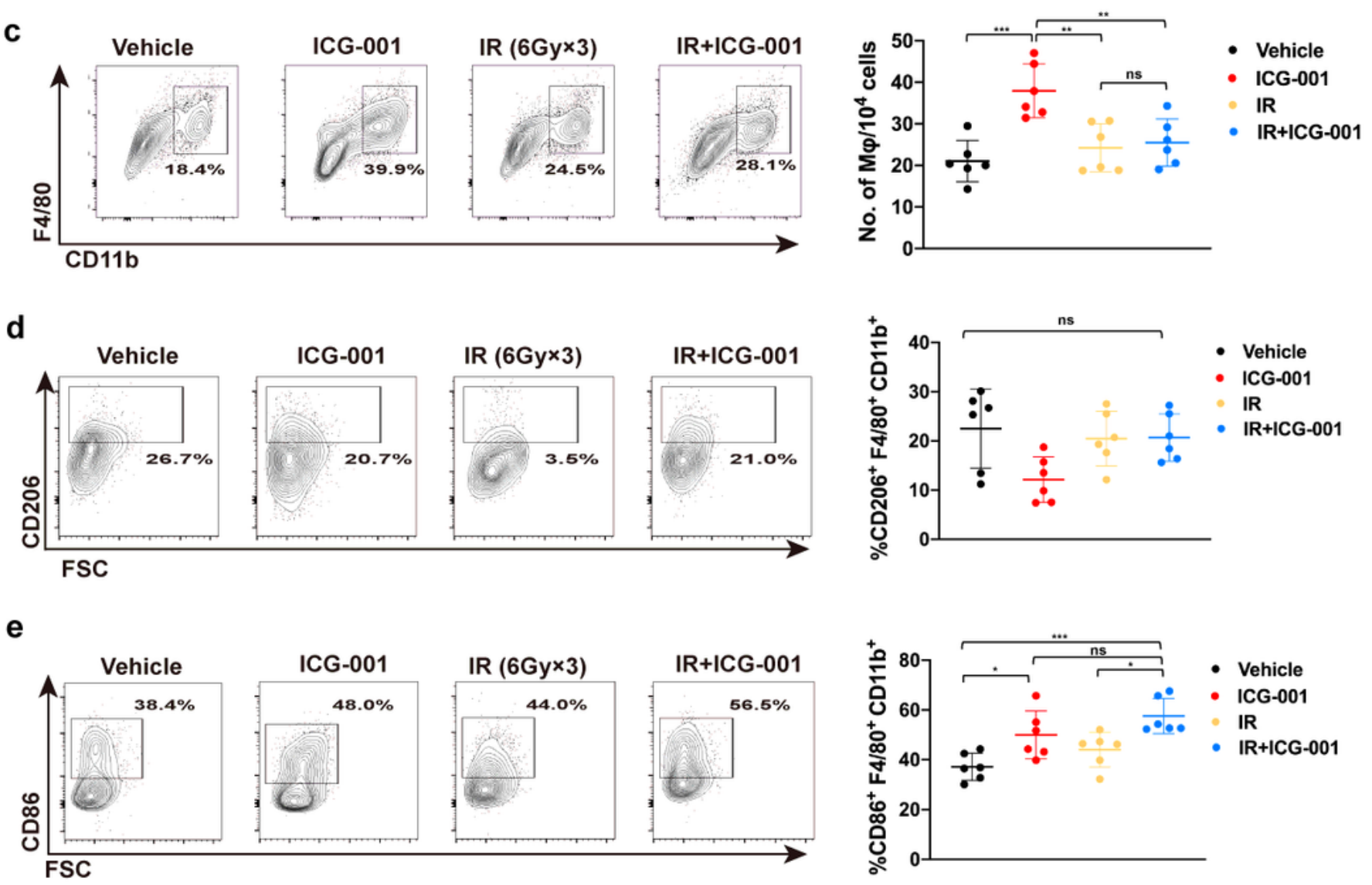

Fig.3

\section{Figure 3}

Combination therapy enhances M1 macrophages infiltration in mice HCC tumors. Representative contour plots and quantitation of DC cells (CD11b- CD45+ CD86+) per 104 cells (a), NK cells (NK1.1+) per 104 cells (b), macrophages (CD11b+ F4/80+) per 104 cells (c), M2 (CD206+)/macrophages ratios (d), and M1 $(\mathrm{CD} 86+) /$ macrophages ratios (e). Data represent the mean $\pm \mathrm{SD}$. Ns, not significant, ${ }^{\star} \mathrm{P}<0.05,{ }^{\star *} \mathrm{P}<0.01$, ***P $<0.001$ via one-way ANOVA with Tukey's multiple comparisons test. 
a

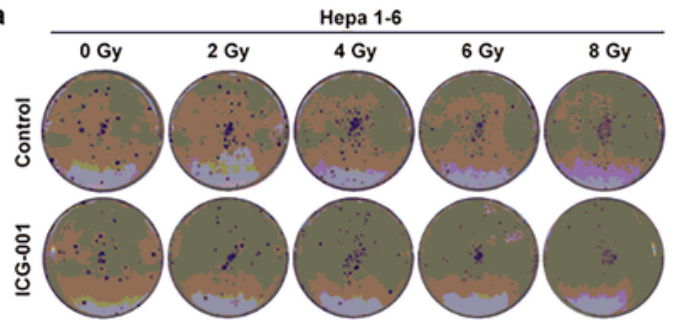

b

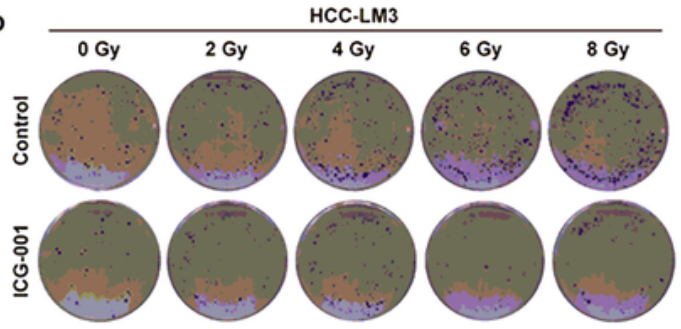

c
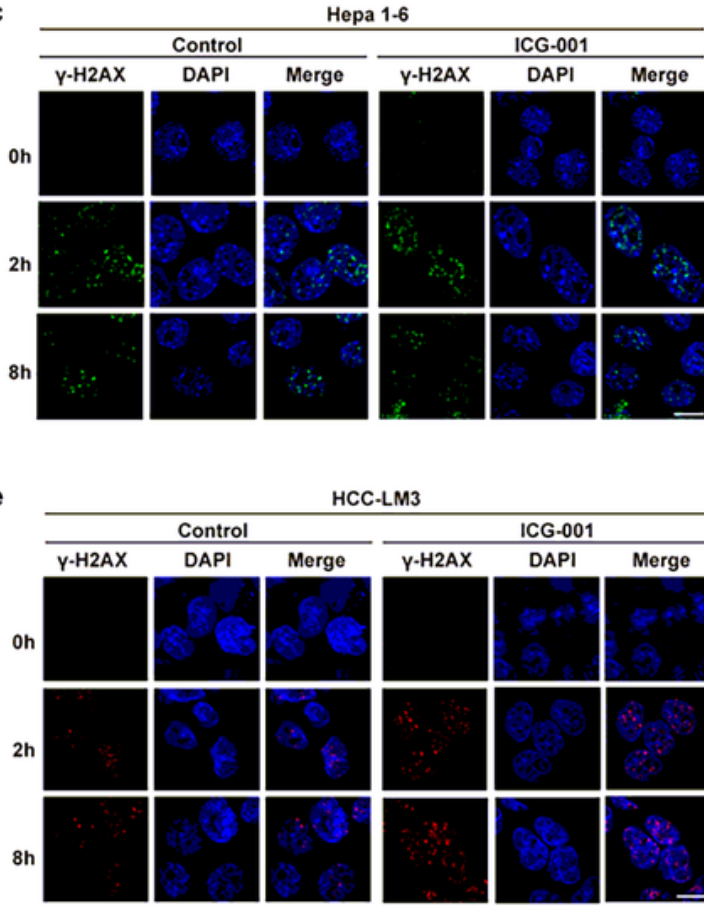

g
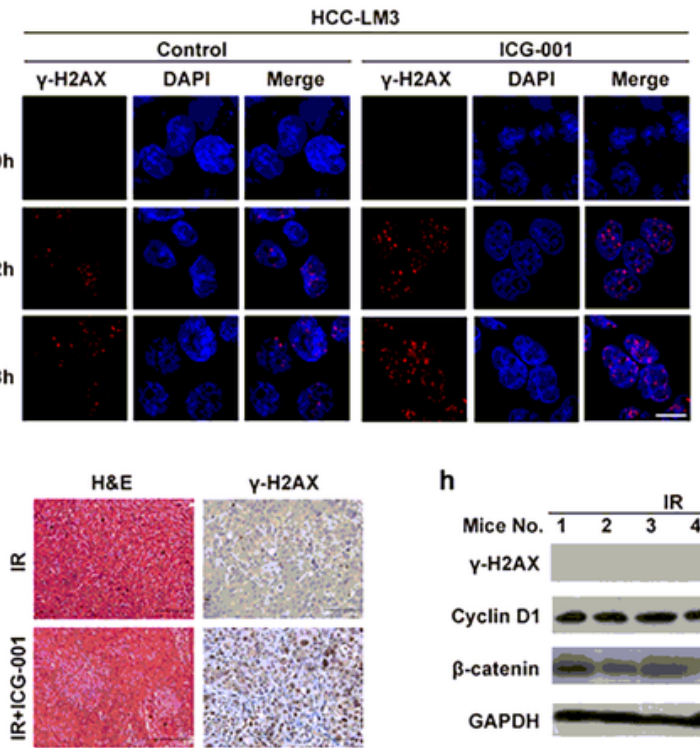
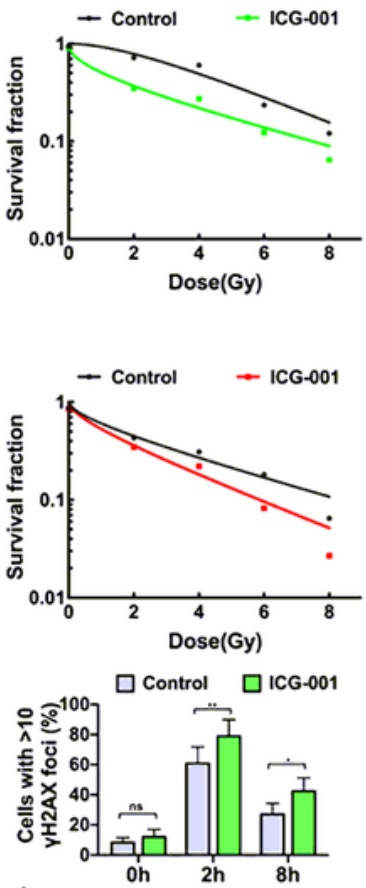

d $\quad \frac{\text { Control }}{0 \mathrm{~h} 2 \mathrm{~h} 8 \mathrm{~h}} \frac{\text { ICG-001 }}{0 \mathrm{~h} 2 \mathrm{~h} 8 \mathrm{~h}}$ $\mathrm{Y} \cdot \mathrm{H} 2 \mathrm{AX}=-0$ c Jun $=--\rightarrow-$ $\beta$-catenin $-1-0$ GAPDH

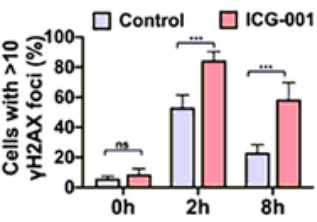

f Control ICG.001 $\mathrm{Y}-\mathrm{H} 2 \mathrm{AX} \longrightarrow \cdots$ c.Jun $=0$ $\beta$-catenin GAPDH $=$

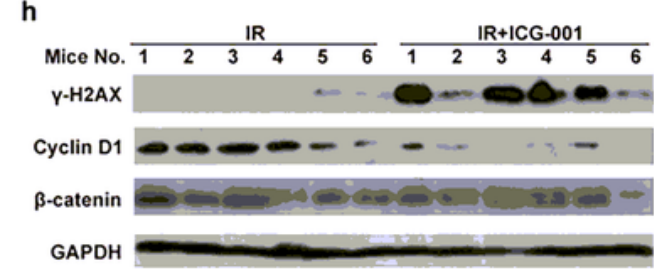

Fig.4

Figure 4

ICG-001 enhances radiosensitivity and radiation-induced DNA damage response in HCC cells. (a-b) Colony formation assays were performed in Hepa1-6 cells (a) or HCC-LM3 cells (b) with pretreatment of ICG-001 (5 $\mu \mathrm{M}, 48 \mathrm{~h})$ before radiation (left panel, representative images of colony formation; right panel,

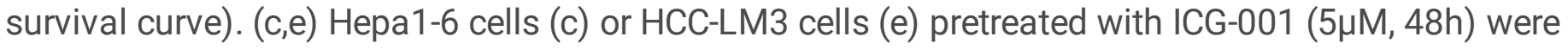
exposed to IR (6 Gy). Cells were collected at the indicated timepoints after IR, and prepared for 
immunofluorescence analysis of $\mathrm{Y}-\mathrm{H} 2 \mathrm{AX}$ nuclear foci. The 0 timepoint indicated no IR. (left panel, representative images; right panel, quantification for the percentage of cells with more than 10 foci from three independent experiments). Scale bar: $20 \mu \mathrm{M}$. Data represent the mean $\pm \mathrm{SD}$. Ns, not significant, ${ }^{*} \mathrm{P}<$ $0.05,{ }^{*} \mathrm{P}<0.01$, and ${ }^{* * * P}<0.001$ by one-way ANOVA with Tukey's multiple comparisons test. $(\mathrm{d}, \mathrm{f})$ Cell lysates from Hepa1-6 cells (d) or HCC-LM3 cells (f) with the same treatment as that in panel $c$ or e were made for immunoblotting of the indicated proteins. GAPDH was used as a loading control. (g) H\&E and IHC staining for $Y-H 2 A X$ of the xenografts from the mice treated with IR or IR+ICG-001. (f) The cells lysates from the xenografts with the same treatment as that in panel $g$ were made for immunoblotting for the indicated proteins. GAPDH was used as a loading control. 
a

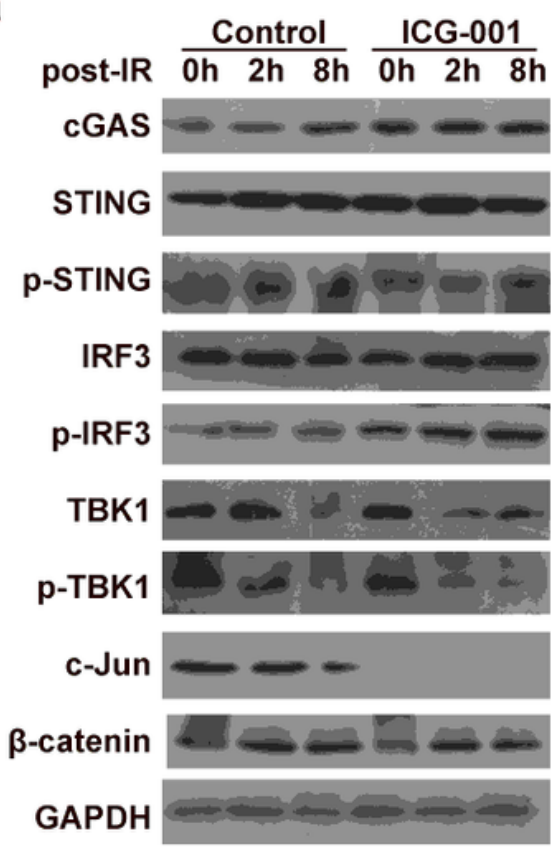

b

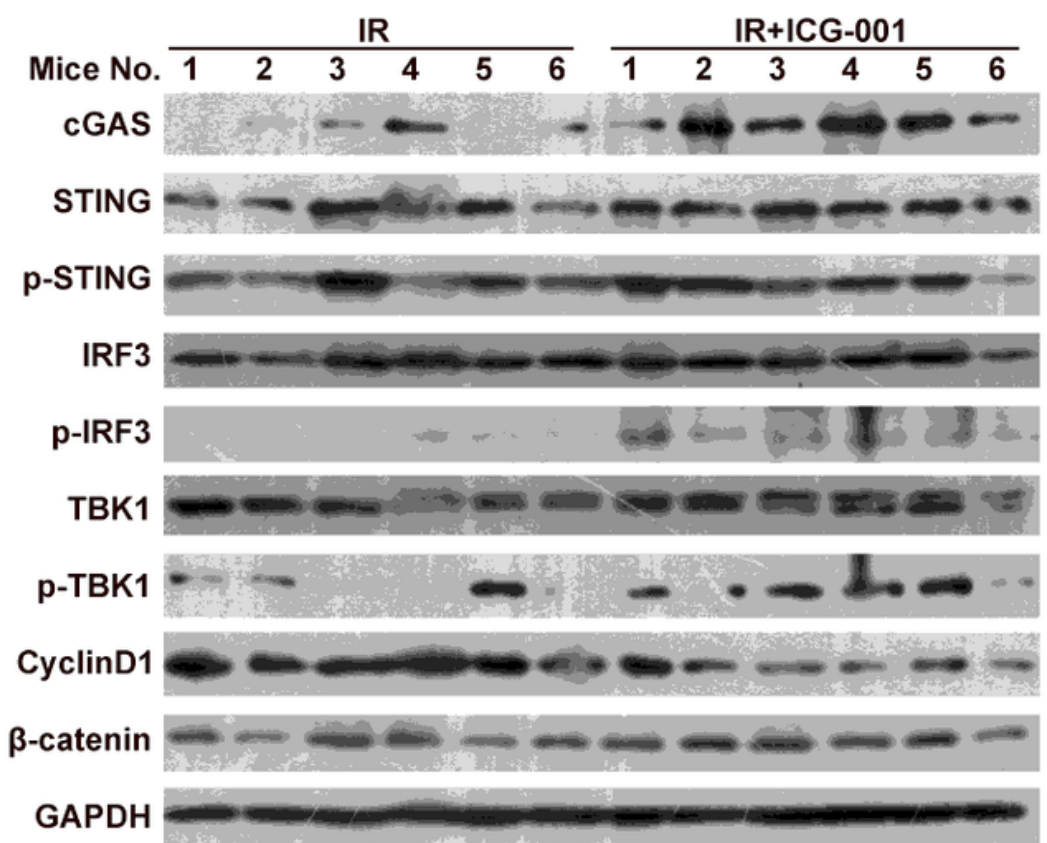

d

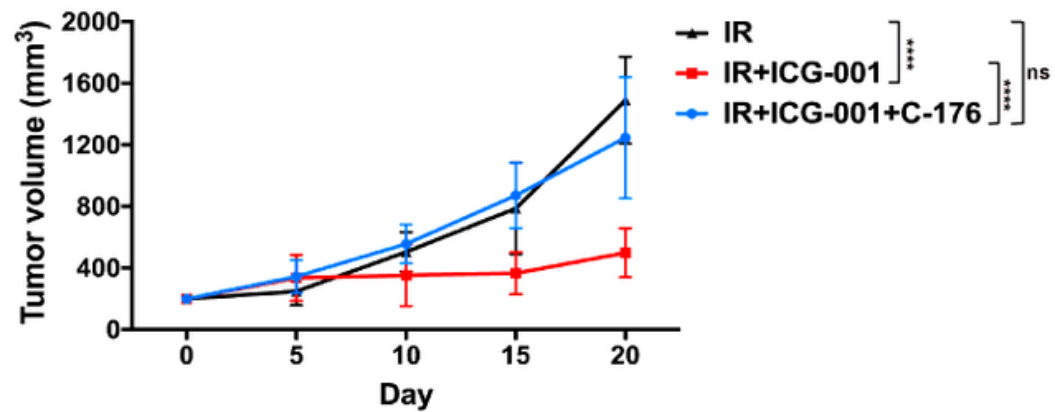

e

- IR IR+C-176

- IR+ICG-001 - IR+ICG-001+C-176

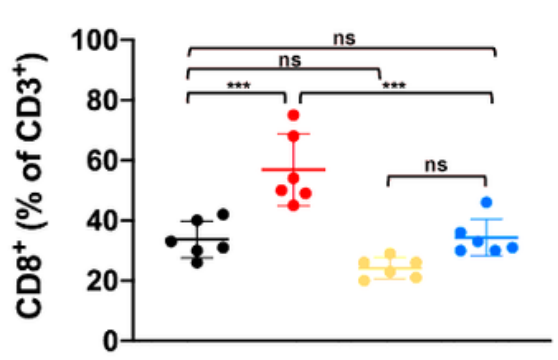

f

- IR

$\mathrm{IR}+\mathrm{C}-176$

- IR+ICG-001 - IR+ICG-001+C-176

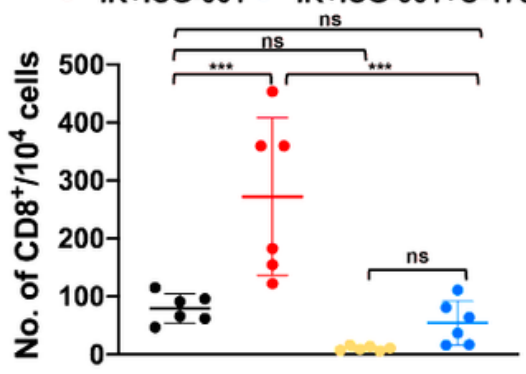

g

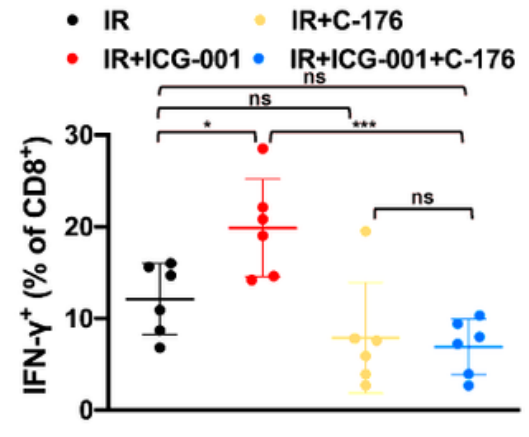

Fig.5

\section{Figure 5}

ICG-001 promotes anti-tumor and TME-improvement effect of radiotherapy in HCC via activating cGAS/STING signaling pathway. (a-b) The key factors of cGAS/STING pathway were detected in Hepa1-6 cells (a) or Hepa1-6 subcutaneous tumors from mice (b) treated with radiotherapy or combination therapy group. (c) Schematic showing schedules of IR, ICG-001, and C-176 treatment. (d) Response of the Hepa1-6 subcutaneous tumors from C57BL/6 mice to IR, IR+ICG-001, and IR+ICG-001+C-176. Data 
represent the mean \pm SD. Statistical analysis was performed by using mixed-effects model, followed by

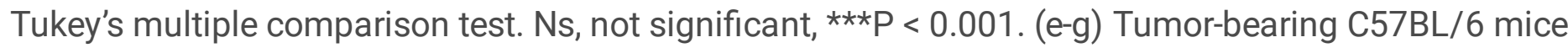
were treated as that shown in panel c, and TILs from the xenografts were isolated and analyzed by flow cytometry. Quantitation of the percentage of TIL CD8+ T cells (e), the number of the TIL CD8+ T cells per 104 cells( $(f)$, and the percentage of TIL CD8 $+T$ cells that elicit IFN- $\gamma$ following stimulation with $P M A / i o n o m y c i n(g)$ were shown. The mean and SD bars shown. Ns, not significant, ${ }^{\star} P<0.05,{ }^{\star} * \mathrm{P}<0.01$, and ${ }^{* *} \mathrm{P}<0.001$ by one-way ANOVA with Tukey's multiple comparisons test.

a

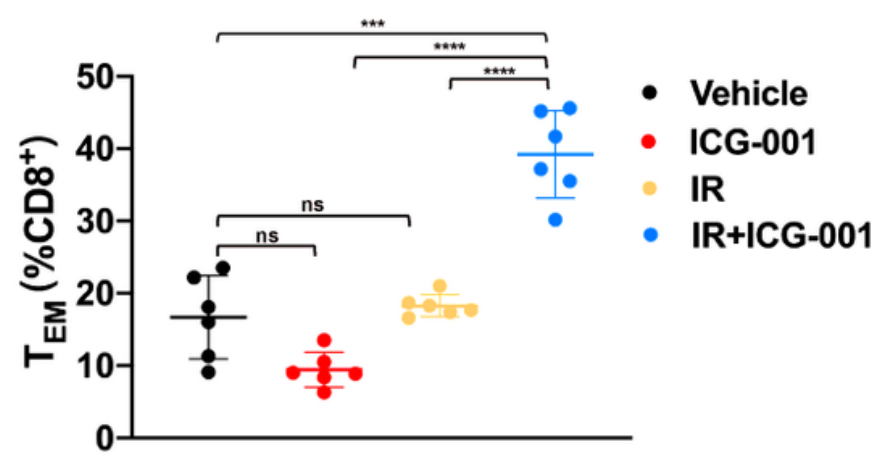

C

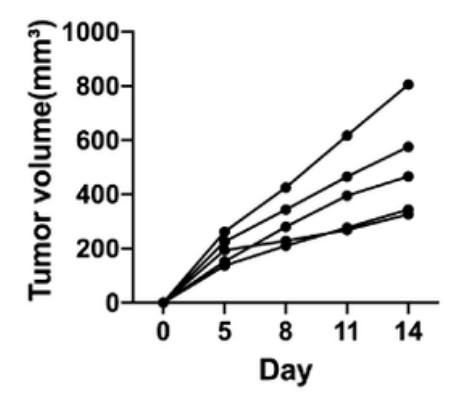

ICG-001

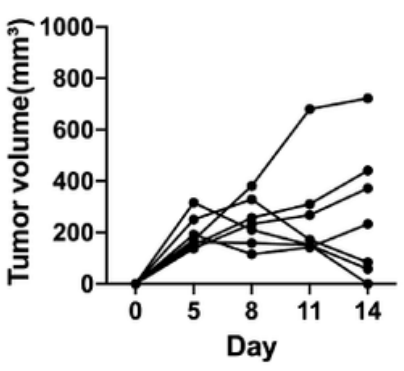

b

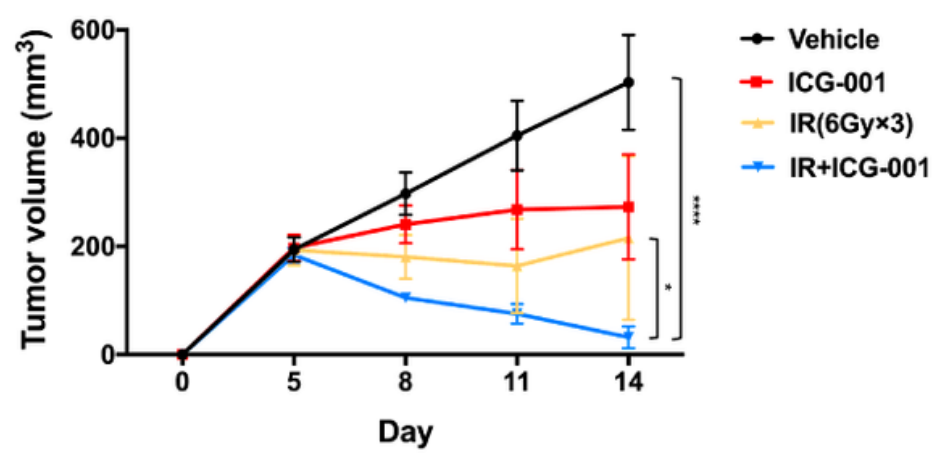

$\operatorname{IR}(6 \mathrm{G} y \times 3)$

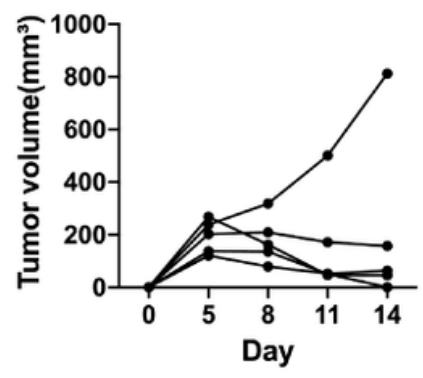

Fig. 6

\section{Figure 6}

Triple therapy enhances immune memory activation in mice. (a) Tumor-bearing C57BL/6 mice were treated as that shown in fig. 1a, and splenic lymphocytes were isolated and analyzed by flow cytometry. Quantitation of the percentage of TEM (CD62L-CD44+) cells was shown. Data represent the mean \pm SD. Ns, not significant, $* \star \star P<0.001$, and ${ }^{* \star \star *} \mathrm{P}<0.0001$ by one-way ANOVA with Tukey's multiple comparisons test. (b-c) C57BL/ 6 mice tumors were re-challenged with Hepal- 6 cells in the contralateral flank and treated with the indicated treatments. The tumor growth curves (b), the individual tumor volume curves (c) were shown. Data represent the mean \pm SD. Statistical analysis was performed using mixedeffects model, followed by Tukey's multiple comparison test. ${ }^{*} \mathrm{P}<0.05, \star \star \star \mathrm{P}<0.001$.

\section{Supplementary Files}

This is a list of supplementary files associated with this preprint. Click to download. 
- SupplementaryFig.docx

- SupplementaryFig.docx 